\title{
Overview of Higgs boson results at ATLAS experiment at the LHC
}

\author{
I.I. Tsukerman on behalf of the ATLAS Collaboration* \\ ITEP, Moscow, 117218, Russia \\ E-mail: ilya.tsukerman@cern.ch
}

One year ago, a new particle with the mass of $125 \mathrm{GeV}$ and properties expected for the Standard Model Higgs boson was discovered by ATLAS and CMS experiments at the Large Hadron Collider at CERN in proton-proton collisions at center-of-mass energies $\sqrt{s}=7 \mathrm{TeV}$ and $8 \mathrm{TeV}$. Since that time more data at $8 \mathrm{TeV}$ were accumulated and analyzed and progress was made in the measurement of the particle properties. In this note an overview of Higgs boson results obtained recently at the ATLAS experiment is given. The complete dataset which corresponds to $4.7 \mathrm{fb}^{-1}$ $\left(20.5 \mathrm{fb}^{-1}\right)$ of the data obtained at $7 \mathrm{TeV}(8 \mathrm{TeV})$ collisions, respectively, is used. The following five signal decay channels are considered: $H \rightarrow \gamma \gamma, H \rightarrow Z Z^{\star} \rightarrow \ell \ell \ell \ell, H \rightarrow W W^{\star} \rightarrow \ell \ell v v$, $H \rightarrow \tau \tau$ and $H \rightarrow b \bar{b}$. These channels are combined to extract the Higgs boson signal mass, strength, coupling constants, spin and parity. All experimental results are compatible with the Standard Model predictions. In addition, upper bounds on the Higgs boson production cross section in rare decay modes $\left(H \rightarrow \mu \mu\right.$ and $\left.H \rightarrow Z \gamma^{\star}\right)$ are obtained.

The XXI International Workshop High Energy Physics and Quantum Field Theory,

June 23 - June 30, 2013

Saint Petersburg Area, Russia

\footnotetext{
* Speaker.
} 


\section{Introduction}

The search for the Standard Model (SM) Higgs boson $(H)$ at the Large Hadron Collider (LHC) [1] is known to be complicated task as expected signal production cross section is $\sigma_{H}=\mathrm{O}(10) \mathrm{pb}$ while background rates are much higher. Fig. 1 (a) shows how predicted $\sigma_{H}$ depends on its mass at different center-of-mass $p p$ energies, $\sqrt{s}=7 \mathrm{TeV}, 8 \mathrm{TeV}$ and $14 \mathrm{TeV}$ [2] which corresponds to the LHC conditions in 2011, 2012 and 2015, respectively. It is about $15 \mathrm{pb}$ at $m_{H}=125 \mathrm{GeV}$ at $7 \mathrm{TeV}$ being 1.3 times higher at $8 \mathrm{TeV}$. At larger $m_{H}$ the cross section increases with $\sqrt{s}$ stronger and the ratio of $8-\mathrm{TeV}$ and $7-\mathrm{TeV}$ rates at $m_{H}=1000 \mathrm{GeV}$ is as large as a factor of four.

There are four main mechanisms of the SM Higgs boson production at LHC energies: gluon fusion ( $g g F$ ) via heavy-quark (mostly top) triangular loop, vector-boson fusion (VBF) where Higgs boson is accompanied by two jets going at small polar angles, associated production with one vector boson ( $V H$, i.e. together with $W$ or $Z$ ) and top-antitop fusion. The first and the last mechanisms as well as the second and the third ones have common couplings ( $t \bar{t} H$ and $V V H$, respectively).

The expected $\sigma_{H}$ from these mechanisms are shown in Fig.1(b) as function of $m_{H}$ at $\sqrt{s}=8$ $\mathrm{TeV}$ together with theoretical uncertainties [2]. They are calculated in the NNLO approximation (except the $t \bar{t} H$ process for which NLO approach is used). It is seen that the ggF mechanism dominates while rates via $\operatorname{VBF}(V H, t \bar{t} H)$ mechanisms are ten (a few dozen, hundred) times smaller, respectively.

The expected branching ratios (BR) of the experimentally favourable decay modes multiplied by $\sigma_{H}$ at $8 \mathrm{TeV}$ are given in Fig.1(c), They are as large as $1.5 \mathrm{pb}$ at $m_{H}=125 \mathrm{GeV}$ for the $H \rightarrow \tau \tau$ and $H \rightarrow W W^{\star} \rightarrow \ell v q \bar{q}$ channels. However, these channels have small signal-to-background (S/B) ratios and poor mass resolution. The $H \rightarrow W W^{\star} \rightarrow \ell v \ell v$ channel has a smaller cross section, 0.25 $\mathrm{pb}$, but one can reach sizeable S/B ratio doing kinematical selections. Again the mass resolution is low. The $H \rightarrow \gamma \gamma$ channel has a very good and simple signature. However, $\sigma_{H} \times B R(H \rightarrow \gamma \gamma)$ is less than $0.05 \mathrm{pb}$, and background conditions are very hard. The cleanest decay mode, where both signature and S/B ratio are perfect is $H \rightarrow Z Z^{\star} \rightarrow \ell \ell \ell \ell$. But $\sigma_{H} \times B R(H \rightarrow \ell \ell \ell \ell)$ is only a few $\mathrm{pb}$ due to the small leptonic branching ratio of the $Z$ boson. Despite all these difficulties, both the ATLAS [3] and CMS [4] Collaborations at the LHC were able to discover one year ago a new particle with the properties of a SM-like Higgs boson combining the $H \rightarrow \gamma \gamma, H \rightarrow Z Z^{\star} \rightarrow \ell \ell \ell \ell$, $H \rightarrow W W^{\star} \rightarrow \ell \ell v v, H \rightarrow b \bar{b}$ and $H \rightarrow \tau \tau$ decay channels. Their studies were based on the complete data taken at $7 \mathrm{TeV}\left(\approx 5 \mathrm{fb}^{-1}\right)$ and on one third of the $8-\mathrm{TeV}$ dataset $\left(\approx 6 \mathrm{fb}^{-1}\right)$. The summary presented in this note mostly uses the complete $7-\mathrm{TeV}$ and $8-\mathrm{TeV}$ ATLAS datasets.

The note is organized as follows. The ATLAS detector is briefly described in Section 2. In Section 3, the most sensitive bosonic decay modes of the SM Higgs boson, namely $H \rightarrow \gamma \gamma$, $H \rightarrow Z Z^{\star} \rightarrow \ell \ell \ell \ell$ and $H \rightarrow W W^{\star} \rightarrow \ell \ell v v$ are considered; Section 4 is devoted to fermionic decay modes like $H \rightarrow \tau \tau$ and $H \rightarrow b \bar{b}$. The results of statistical combination of these five channels are presented in Section 5. This combination includes such properties as mass, signal strength, coupling constants, spin and parity. Upper bounds on the SM-like Higgs boson production cross section in rare decay modes such as $H \rightarrow \mu \mu$ and $H \rightarrow Z \gamma^{\star}$ are given in Section 6. Conclusions are drawn in Section 7. 


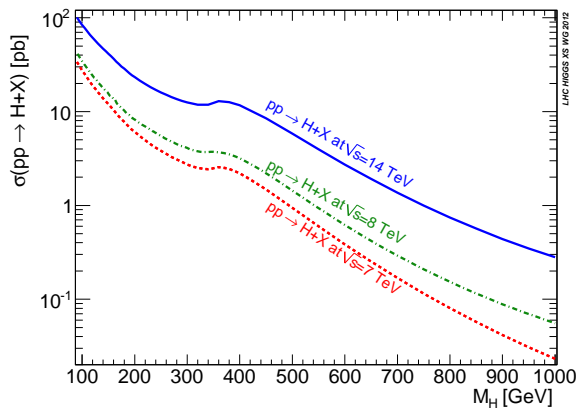

(a)

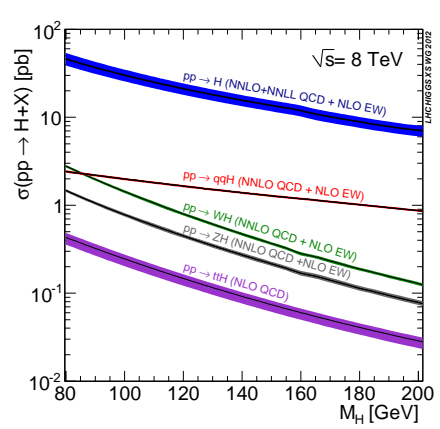

(b)

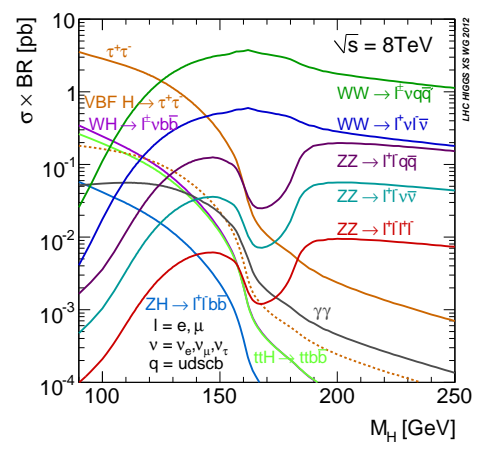

(c)

Figure 1: (a) Predicted total $\sigma_{H}$ at $\sqrt{s}=7,8$ and $14 \mathrm{TeV}$ as function of $m_{H}$ [2]. (b) Predicted total $\sigma_{H}$ at $\sqrt{s}=8 \mathrm{TeV}$ together with separate contributions from different production mechanisms as function of $m_{H}$ [2]. (c) $\sigma_{H} \times B R(H \rightarrow X)$ at $\sqrt{s}=8 \mathrm{TeV}$ for the most promising decay channels $X$ as function of $m_{H}$ [记].

\section{The ATLAS detector and its modeling}

The ATLAS detector [5] is a multipurpose particle physics apparatus with forward-backward symmetric cylindrical geometry. The inner tracking detector (ID) consists of a silicon pixel detector, a silicon microstrip detector, and a straw-tube transition radiation tracker. The ID is surrounded by a thin superconducting solenoid which provides a $2 \mathrm{~T}$ magnetic field, and by high-granularity liquid-argon (LAr) sampling electromagnetic (EM) calorimetry. The electromagnetic calorimeter is divided into a central barrel and end-cap regions on either end of the detector. In the region matched to the ID $\left(|\eta|<2.5^{1}\right)$, it is radially segmented into three layers. The first layer has a fine segmentation in $\eta$ to facilitate $\mathrm{e} / \gamma$ separation from $\pi^{0}$ and to improve the resolution of the shower position and direction measurements. In the region $|\eta|<1.8$, the electromagnetic calorimeter is preceded by a presampler detector to correct for upstream energy los ses. An iron-scintillator/tile calorimeter gives hadronic coverage in the central rapidity range $(|\eta|<1.7)$, while a LAr hadronic end-cap calorimeter provides coverage over $1.5<|\eta|<3.2$. The forward regions $(3.2<|\eta|<4.9)$ are instrumented with LAr calorimeters for both electromagnetic and hadronic measurements. The muon spectrometer (MS) surrounds the calorimeters and consists of three large air-core superconducting magnets providing a toroidal field, each with eight coils, a system of precision tracking chambers, and fast detectors for triggering. The combination of all these systems provides charged particle measurements together with efficient and precise lepton and photon measurements in the pseudorapidity range $|\eta|<2.5$. Jets and missing transverse energy, $E_{T}^{\text {miss }}$, are reconstructed using energy deposits over the full coverage of the calorimeters, $|\eta|<4.9$.

To develop analysis procedures, to calculate signal (S) acceptance and to evaluate the contri-

\footnotetext{
${ }^{1}$ ATLAS uses a right-handed coordinate system with the origin at the nominal interaction point (IP) in the centre of the detector, and the $z$-axis along the beam line. The $x$-axis points from the IP to the centre of the LHC ring, and the $y$-axis points upwards. Cylindrical coordinates $(r, \theta)$ are used in the transverse plane, $\theta$ being the azimuthal angle around the beam line. Observables labelled "transverse" are projected into the $x-y$ plane. The pseudorapidity is defined in terms of the polar angle $\theta$, as $\eta=\ln \tan (\theta / 2)$.
} 
bution for some background (B) processes, Monte Carlo (MC) modeling is used. All simulated signal and background datasets are passed through a GEANT4 [6] full simulation chain of the ATLAS set-up and algorithms used also in real data recontruction. Cross sections are calculated at the NLO (or in some cases at the NLO+NLL/NNLL) level. ${ }^{2}$ Multiple interactions in the same bunch crossing ${ }^{3}$ including detector effects are also modeled.

In many cases backgrounds are directly measured experimentally in so-called control regions (CR), kinematically orthogonal to signal regions (SR). The corresponding normalization factors are determined with simulated MC samples.

\section{3. $H \rightarrow \gamma \gamma, H \rightarrow Z Z^{\star} \rightarrow \ell \ell \ell \ell$ and $H \rightarrow W W^{\star} \rightarrow \ell \ell v v$ decay channels}

The results of analyses of complete ATLAS dataset at $\sqrt{s}=7 \mathrm{TeV}$ and $8 \mathrm{TeV}$ are presented below. They are summarized mostly in Ref.[7] and in Ref.[8].

\section{1 $H \rightarrow \gamma \gamma$}

The expected cross section of $H \rightarrow \gamma \gamma$ production and decay is $30-50 \mathrm{fb}$ in the region $m_{H}=110$ $150 \mathrm{GeV}$. At higher Higgs boson masses it falls rapidly mainly due to the strong decrease of the $\mathrm{BR}(H \rightarrow \gamma \gamma)$. The signature is two isolated photons with invariant mass equal to $m_{H}$. So excellent energy and angular resolutions are important. Simulations show that the ATLAS EM calorimeter allows to reach invariant-mass resolution $F W H M \approx 4 \mathrm{GeV}$ in the region of interest. Irreducible background consists of continuum production of photon pairs via $q \bar{q}+g g \rightarrow \gamma \gamma$ mechanisms. These processes have about a hundred times larger rates than expected for the signal. Reducible backgrounds include mostly $\gamma$ jet- and jet jet-production when one or two jets are misidentified as photons in the detector. These processes have many orders of magnitude higher cross sections than the continuum diphoton production. Thanks to the EM calorimeter with a preshower detector, one can reach jet rejection at the level of $10^{4}$. After kinematical selections $75-80 \%$ of observed $\gamma \gamma$ events have both real photons while only about $20 \%(\leq 5 \%)$ events contain one (two) misidentified jets, respectively [ $[$ ]. Some $13 \mathrm{~K}$ events survive all the selections while the expected signal in the mass window around $125 \mathrm{GeV}$ is $\approx 400$. To increase the discovery potential, the events are further subdivided into fourteen independent categories having different expected $m_{\gamma \gamma}$ resolution and $\mathrm{S} / \mathrm{B}$ ratio. The $m_{\gamma \gamma}$-distribution after the corresponding reweighting is given in Fig. (2a) together with the spectrum after background subtraction. An excess of events is seen around $126 \mathrm{GeV}$. The probability that background fluctuation causes at least the observed excess of events, called $p_{0}$, is shown in Fig.2(b) as a function of $m_{H}$. A clear signal at around $126.5 \mathrm{GeV}$ with $7.4 \sigma$ local significance is seen. The measured mass of the new particle is $126.8 \pm 0.2$ (stat) \pm 0.7 (syst) GeV, where the systematic error is mainly due to the photon energy scale uncertainty. Taking into account the known Landau-Yang theorem, this particle cannot have spin equal to one.

Fig.(a) shows the signal strength in units of the SM for $V H$, VBF and $g g F+t \bar{t} H$ couplings separately, and the combined one. The measured values are the following: $\mu_{g g F+t \bar{t} H} \times B / B_{S M}=1.6_{-0.3}^{+0.3}(\text { stat })_{-0.2}^{+0.3}(\mathrm{syst}) ; \mu_{V B F} \times B / B_{S M}=1.7_{-0.8}^{+0.8}(\text { stat })_{-0.4}^{+0.5}(\mathrm{syst}) ;$

\footnotetext{
${ }^{2}$ Most of event generators work at the NLO level; higher order effects are simulated with parton showers.

${ }^{3}$ On average, approximately 10 (20) interactions occurred each $50 \mathrm{~ns}$ in the same bunch crossing at $\sqrt{s}=7 \mathrm{TeV}(8$ $\mathrm{TeV})$.
} 
$\mu_{V H} \times B / B_{S M}=1.8_{-1.3}^{+1.5}$ (stat) ${ }_{-0.3}^{+0.3}$ (syst). Combining them one obtains a value $\mu=1.65 \pm 0.24$ (stat) ${ }_{-0.18}^{+0.25}$ (syst) which is $2.3 \sigma$ away from the SM prediction of unity.

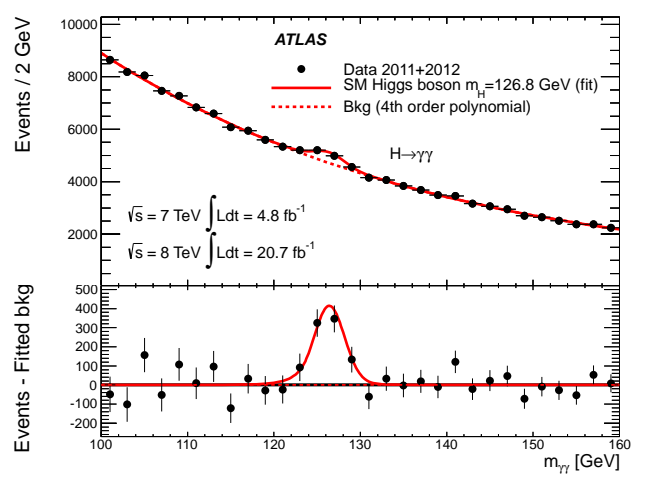

(a)

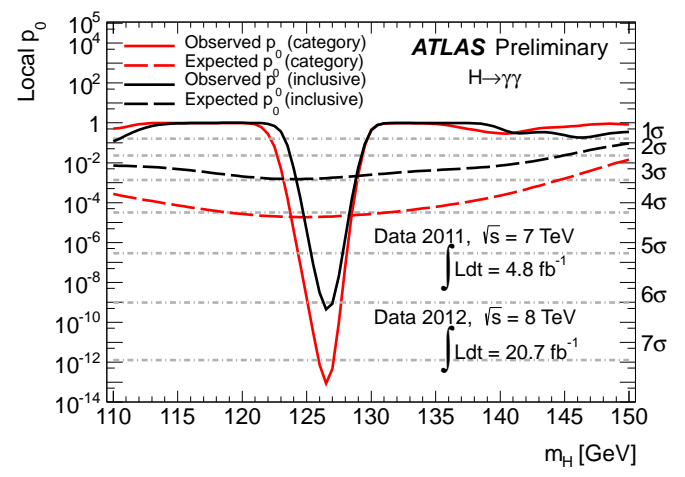

(b)

Figure 2: Results of the $H \rightarrow \gamma \gamma$ study. (a) $m_{\gamma \gamma}$-distribution of diphoton candidates after all selections of the inclusive analysis for the combined $7 \mathrm{TeV}$ and $8 \mathrm{TeV}$ data 仍. The result of a fit to the data with the sum of a SM Higgs boson signal (with $m_{H}=126.8 \mathrm{GeV}$ and free signal strength) and background is superimposed. The residuals of the data with respect to the fitted background are displayed in the lower panel. (b) The observed local $p_{0}$ value as function of $m_{H}$ for the combination of $7-\mathrm{TeV}$ and $8-\mathrm{TeV}$ data for the inclusive case (black) and for the analysis using categories (red) [10].

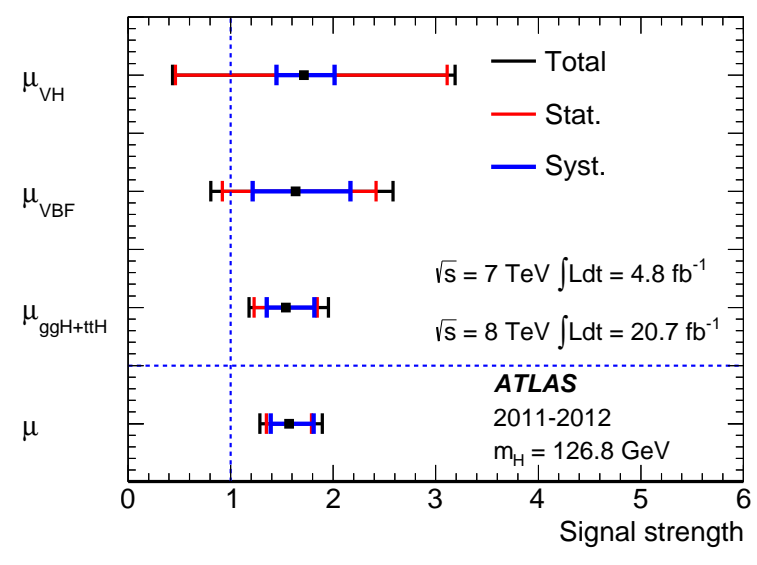

(a)

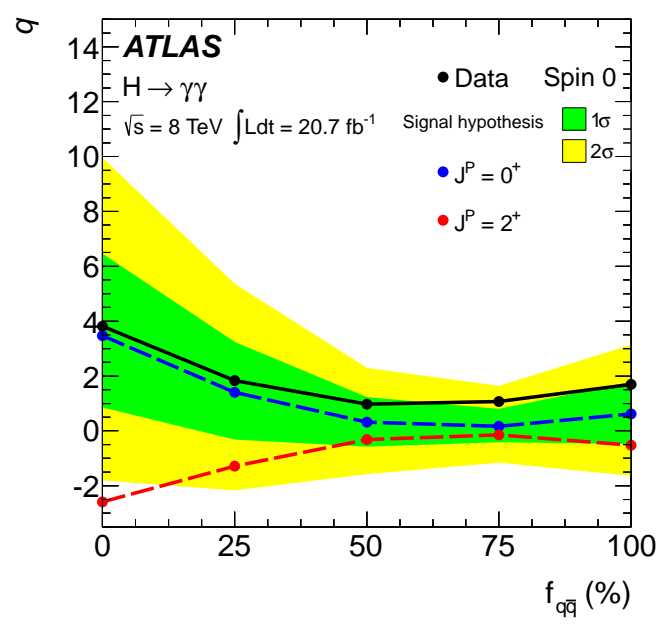

(b)

Figure 3: Results of the $H \rightarrow \gamma \gamma$ study. (a) Measured signal strengths $\mu(g g F+t t H), \mu(V B F)$ and $\mu(V H)$

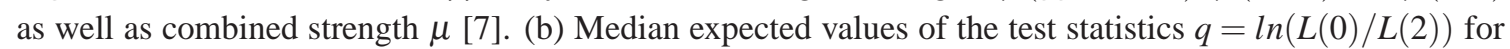
the spin- 0 (dashed blue/upper line and corresponding $68 \%$ and $95 \%$ CL bands) and spin-2 (dashed red/lower line) hypotheses, as well as the observed values (solid line) as function of the $f_{q \bar{q}}$ [ 8$]$.

In addition, the fiducial cross section times branching ratio to the two photon decay, defined for the kinematic range $E_{T}^{\gamma 1} \geq 40 \mathrm{GeV}, E_{T}^{\gamma 2} \geq 30 \mathrm{GeV}$, and $|\eta|^{\gamma} \leq 2.37$, and measured using 20.7 
$\mathrm{fb}^{-1}$ data at $\sqrt{s}=8 \mathrm{TeV}$ is $56.2 \pm 10.5$ (stat) \pm 6.5 (syst) \pm 2.0 (lumi) fb.

Using the distribution of $\cos \theta^{\star}$ in the so-called Collins-Soper frame one can exclude a possibility of spin- 2 for the newly observed particle. The obtained log-likelihood ratio of spin- 0 and spin-2 hypotheses is given in Fig. 3(b) as function of $f_{q \bar{q}}=\sigma_{H}^{q \bar{q}} / \sigma_{H}^{\text {total }}$. One can see that the spin-2 hypothesis ${ }^{4}$ is excluded at $99 \% \mathrm{CL}$ assuming that all signal events are produced through $g g$-fusion. This number is reduced to $95 \%$ if a $25 \%$ admixture of $q \bar{q}$ events is supposed.

\section{2 $H \rightarrow Z Z^{\star} \rightarrow \ell \ell \ell \ell$}

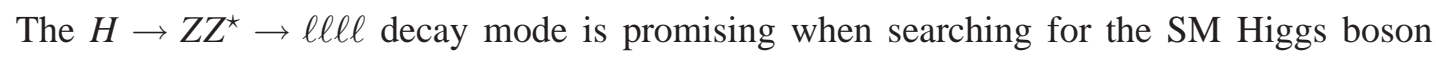
in a wide mass range from $110 \mathrm{GeV}$ to $600 \mathrm{GeV}$. The $\sigma_{H} \times B R(H \rightarrow \ell \ell \ell \ell)$ is $3 \mathrm{fb}$ at $m_{H}=125$ $\mathrm{GeV}$ for $\sqrt{s}=8 \mathrm{TeV}$. The signal signature is two pairs of isolated, opposite-sign leptons (electrons or muons); at least one pair should have $m_{\ell \ell}$ close to $m_{Z}$. Irreducible backgrounds come from $q \bar{q}+g g \rightarrow Z Z^{\star}$ processes which have comparable cross sections to the expected signal at 125 $\mathrm{GeV}$. Smaller reducible backgrounds are due to $Z+j e t s$ and $t \bar{t}$ production. The ATLAS detector has excellent four-lepton invariant-mass resolution to see a narrow and small signal peak, namely $1.6 \mathrm{GeV}(1.9 \mathrm{GeV}, 2.4 \mathrm{GeV})$ for the $4 \mu(2 \mu 2 e, 4 e)$ cases, respectively. Measured invariant-mass distribution, $m_{4 \ell}$, after combination of all lepton cases is shown in Fig.t(a). A clear maximum above background is seen in the region around $125 \mathrm{GeV} ; 32$ events are observed in the mass window $125 \pm 5 \mathrm{GeV}$ with an estimated background $11.1 \pm 1.0$ events. The data/MC ratio is compatible with unity in the sidebands. The peak at $m_{4 \ell}=m_{Z}$ is due to the rare $Z$ boson decay to four leptons.

The $p_{0}$ probability is shown in Fig.t(b) as a function of $m_{H}$ in the region $110-180 \mathrm{GeV}$. The local significance reaches $6.6 \sigma$ at $124 \mathrm{GeV}$ and no other big fluctuations are observed in the mentioned mass range. The reconstructed mass of the new particle is $124.3 \pm 0.6$ (stat) \pm 0.4 (syst) $\mathrm{GeV}$. The measured signal strength, $\mu=1.7 \pm 0.4$, is compatible with the SM prediction of one at the $2 \sigma$ level.

To search for a Higgs-like boson in addition to the one seen at $124.3 \mathrm{GeV}$, the mass distribution above $m_{4 \ell}=200 \mathrm{GeV}$ is analysed. For this analysis, the event classification into ggF-like, VBFlike and VH-like categories is employed. A SM-like Higgs boson is excluded at 95\% CL with the mass below about $650 \mathrm{GeV}(350 \mathrm{GeV})$ in the $\mathrm{ggF}(\mathrm{VBF}+\mathrm{VH})$ mechanisms alone [11].

Despite small signal statistics, the excellent $\mathrm{S} / \mathrm{B}$ ratio allows to study such properties of the signal as spin and parity using angular distributions of the newly observed particle's decay products, see Fig.5(a). Five spin-parity hypotheses are tested: $0^{+}$(as predicted by the SM), $0^{-}, 1^{+}, 1^{-}$and $2^{+}$. Events passing all kinematical selections having $115 \mathrm{GeV} \leq m_{4 \ell} \leq 130 \mathrm{GeV}$ are used in this study. The analysis is based on a Boosted Decision Tree (BDT). As an example, Fig 5(b) shows distributions of log-likelihood ratios for the $0^{+}$and $0^{-}$hypotheses.

The analysis has shown that the $1^{+}$hypothesis is strongly disfavoured with respect to the $0^{+}$ hypothesis. However, exclusion of the $0^{-}, 1^{-}$and $2^{+}$cases is less effective. One can conclude that $0^{-}\left(1^{-}, 1^{+}\right)$states are excluded at $\mathrm{CL}=97.8 \%(94 \%, 99.8 \%)$ using the $\mathrm{CL}_{s}$ method in favour of the $0^{+}[8]$. The $0^{+}$and $2^{+}$hypotheses are also compared assuming different $f_{q \bar{q}}$ fraction. The expected separation does not depend on this fraction in this channel.

\footnotetext{
${ }^{4}$ Graviton-like model is used to simulate the spin-2 case.
} 


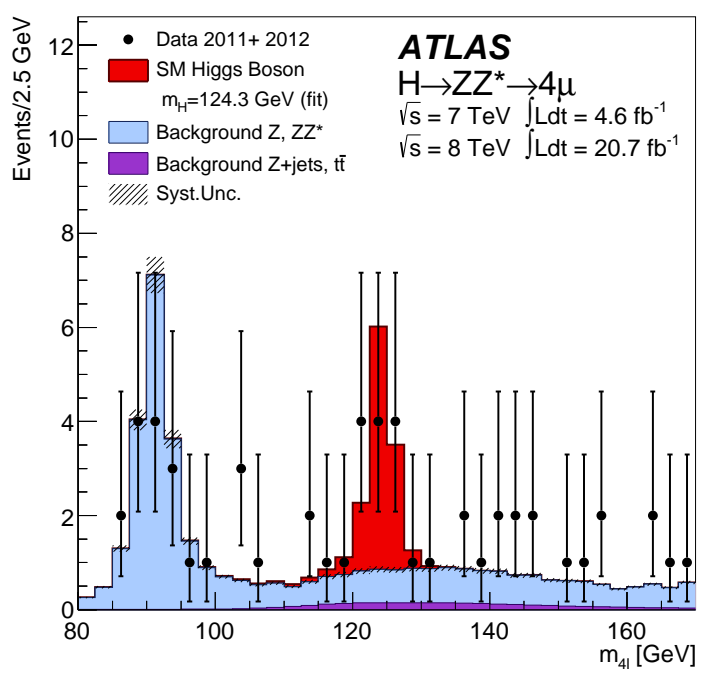

(a)

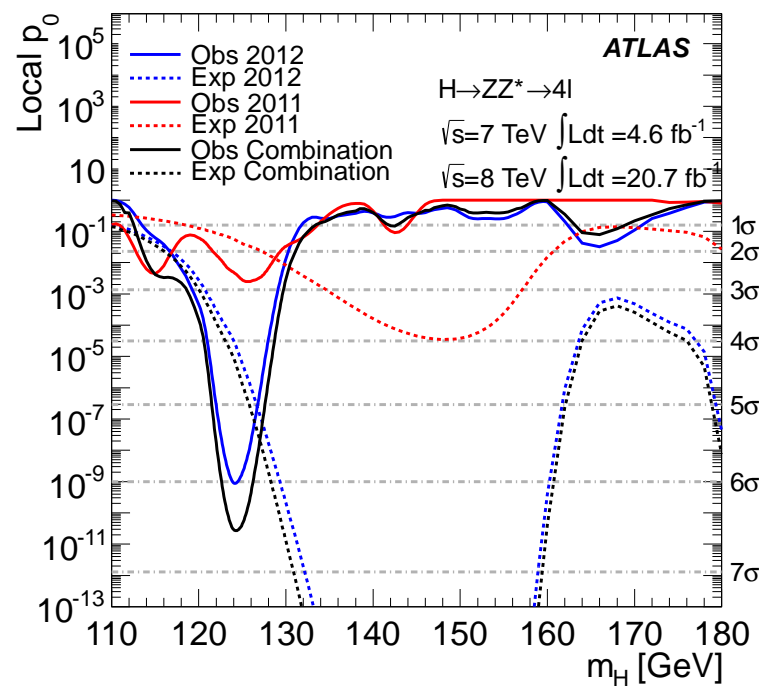

(b)

Figure 4: Results of the $H \rightarrow Z Z^{\star} \rightarrow 4 \ell$ study [7]. (a) $m_{4 \ell}$ distributions for the selected candidates compared to the background expectation in the mass range $80-170 \mathrm{GeV}$. The signal expectation for the $m_{H}=125 \mathrm{GeV}$ hypothesis is also shown. (b) The observed local $p_{0}$-value for the combination of the 2011 and 2012 data sets (solid black line); the $\sqrt{(s)}=7 \mathrm{TeV}$ and $8 \mathrm{TeV}$ data results are shown in solid lines (blue and red, respectively).

\section{3 $H \rightarrow W W^{\star} \rightarrow \ell \ell v v$}

$H \rightarrow W W^{\star} \rightarrow \ell \ell v v$ decay channel has an expected cross section of about $250 \mathrm{fb}$ at $8 \mathrm{TeV}$ for $m_{H}=125 \mathrm{GeV}$. The search mass range is $110-200 \mathrm{GeV}$. The signature is two isolated oppositesign leptons (electrons or muons) and $E_{T}^{m i s s}$ due to neutrinos so one cannot reconstruct the Higgs boson mass. Instead, its transverse mass reconstruction is possible. It is calculated as $m_{T}=$ $\sqrt{\left(E_{T}^{\ell \ell}+E_{T}^{m i s s}\right)^{2}-\left|\mathbf{P}_{\mathbf{T}}^{\ell \ell}+\mathbf{E}_{\mathbf{T}}^{\text {miss }}\right|^{2}}$, where $E_{T}^{\ell \ell}=\sqrt{\left|P_{T}^{\ell \ell}\right|^{2}+m_{\ell \ell}^{2}}$ and $P_{T}^{\ell \ell}\left(m_{\ell \ell}\right)$ are transverse momentum (invariant-mass) of the di-lepton system, respectively. So knowledge of background normalization and shapes is of importance. Backgrounds come from $q \bar{q}+g g \rightarrow W W$ continuum production as well as from $t \bar{t}, W t$ and $Z+$ jets processes with a di-lepton final state. $W+$ jets with a jet misidentified as a lepton has also a sizeable rate. Separate CR for each of these backgrounds are defined to measure them. The event sample is subdivided in accordance with the number of jets $(0,1$ or $\geq 2)$ and in accordance with di-lepton flavour $(e e, \mu \mu$ or $e \mu)$. For the 0 and 1 jet case the ggF mechanism is expected to be dominant while for the multi-jet case signal events are mostly due to the VBF mechanism. The $m_{T}$-distribution after background subtraction is shown in Fig.6(a). A clear excess of events is seen in the region $80-140 \mathrm{GeV}$. The local $p_{0}$ as a function of $m_{H}$ is shown in Fig.6(b). It corresponds to $3.8 \sigma$ signal significance at $126 \mathrm{GeV}$. The fitted signal strength is equal to $1.0 \pm 0.3$, i.e. compatible with the SM prediction. 95\% CL of signal exclusion is reached (expected) in the region above 134 (118) GeV. Spin properties are also studied with the $H \rightarrow W W^{\star} \rightarrow \ell \ell v v$ decay channel [8]. They are not discussed in this note. 


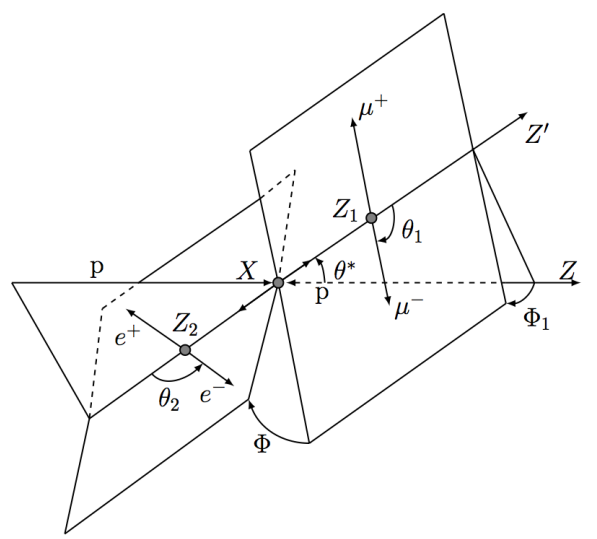

(a)

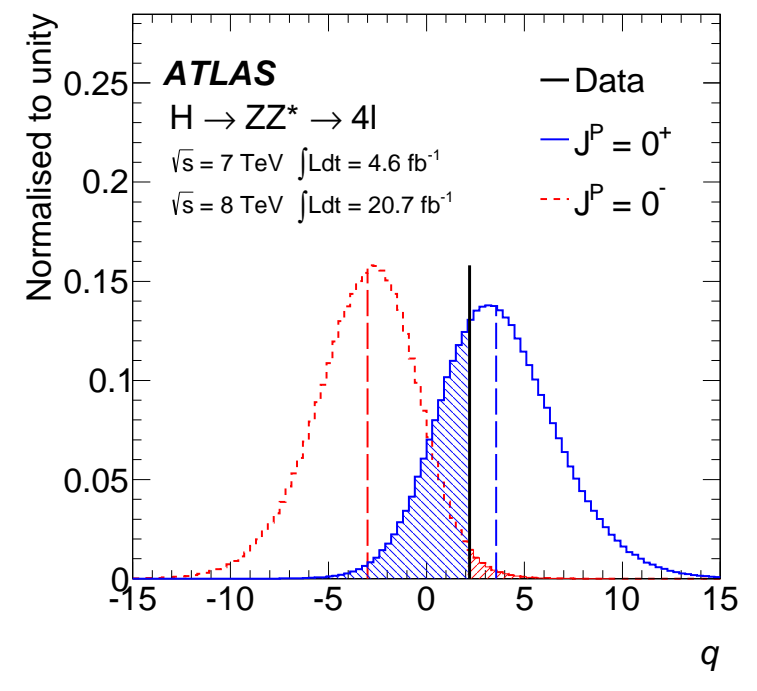

(b)

Figure 5: (a) Definition of the production and decay angles in an $H \rightarrow Z Z^{\star} \rightarrow \ell \ell \ell \ell$ decay. The illustration is drawn with the beam axis in the lab frame, $Z_{1}$ and $Z_{2}$ in the $\mathrm{X}$ rest frame and the leptons in their corresponding parent rest frames (see [11] for further description). (b) Expected distributions of $q=\log \left(L\left(0^{+}\right) / L\left(0^{-}\right)\right)$, the logarithm of the ratio of profiled likelihoods, under the hypotheses for the $\mathrm{SM}^{+}$(blue/solid line distribution) or $0^{-}$(red/dashed line distribution) signals [8]. The observed value is indicated by the vertical solid line and the expected medians by the dashed lines. The coloured areas correspond to the integrals of the expected distributions up to the observed value and are used to compute the $p_{0}$-values for the rejection of each hypothesis.

\section{4. $H \rightarrow \tau \tau$ and $H \rightarrow b \bar{b}$ decay channels}

Results based on the complete 7-TeV dataset and $60 \%$ of $8-\mathrm{TeV}$ dataset are briefly discussed in this section.

\section{1 $H \rightarrow \tau \tau$ decay channel [13]}

The search is performed in the region $100-150 \mathrm{GeV}$, where $B R(H \rightarrow \tau \tau)$ is reasonably high. The signature is two opposite-sign reconstructed isolated $\tau$-leptons with $m_{\tau \tau}$ close to $m_{H}{ }^{5}$ The data sample is divided in accordance with number of leptons from $\tau$ decays $(0,1$ and 2$)$ and number of jets. Backgrounds include $Z^{\star} / \gamma^{\star} \rightarrow \tau \tau, t \bar{t}$-, Wt- and diboson production with the same final state as the signal.

From Fig.7(a) one can see that obtained upper limits on the $\sigma_{H} \times B R(H \rightarrow \tau \tau)$ lie between 1.8 and 3.3 times the SM prediction while expected values are between 1.1 and 3.2. Observed local significance is $1.1 \sigma$ at $m_{H}=125 \mathrm{GeV}$ which corresponds to signal strength of $0.7 \pm 0.7$ in units of the SM.

\footnotetext{
${ }^{5}$ The mass is reconstructed in the so-called collinear approximation, i.e. assuming that neutrinos from $\tau$ decays go in the same direction as the $\tau$ themselves.
} 


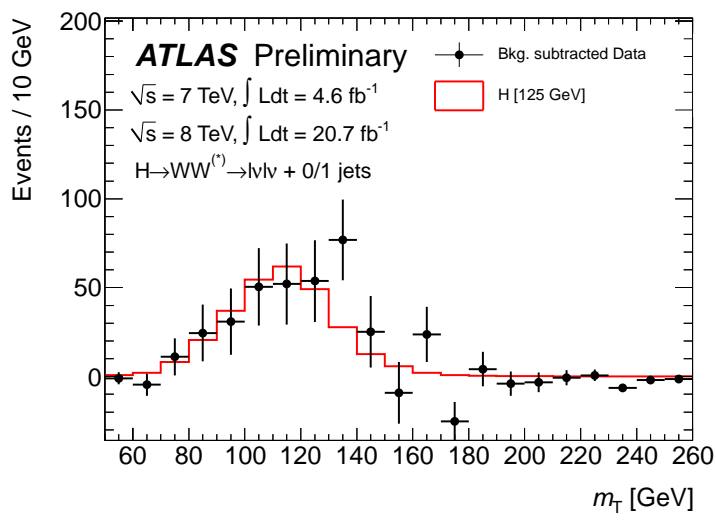

(a)

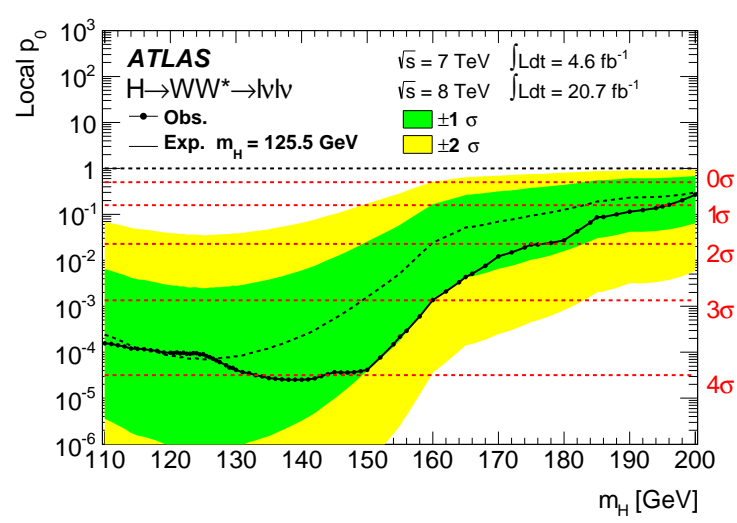

(b)

Figure 6: Results of the $H \rightarrow W W^{\star} \rightarrow \ell \ell v v$ study [7]. (a) Background-subtracted $m_{T}$ distribution. The signal is overlaid. The error bars represent the statistical uncertainties of the data and the subtracted background; it does not include the systematic uncertainties of the latter. (b) Results for $p_{0}$ as a function of $m_{H}$. The smaller green bands represent $1 \sigma$ uncertainties on the expected values, and the larger yellow bands represent $2 \sigma$ uncertainties.

Very recently a new analysis with the complete ATLAS dataset was finished and results are published in Ref.[14].

\section{2 $H \rightarrow b \bar{b}$ decay channel [15]}

Among all channels, $H \rightarrow b \bar{b}$ is predicted to have the largest rate in the mass range 110-130 $\mathrm{GeV}$. However, background is known to be huge. For this reason, here associated $V H$-production is of interest. The signature includes two jets originating from $b$-quarks having an invariant mass close to $m_{H}$ as well as one or two leptons or $E_{T}^{\text {miss }}$ depending on the decay mode of the associated vector boson ( $W \rightarrow \ell v, Z \rightarrow \ell \ell, Z \rightarrow v v$, respectively). Background consists of $W / Z+$ jets, dibosons, top and multijet production with fake leptons. The $95 \% \mathrm{CL}$ exclusion limit lies between $1.0 \sigma_{S M}$ and $2.0 \sigma_{S M}$ in the $m_{H}$ range between $110 \mathrm{GeV}$ and $130 \mathrm{GeV}$ being $1.7 \sigma_{S M}$ at $125 \mathrm{GeV}$, see Fig. (7)(b).

Recently, preliminary results of a new analysis based on complete dataset were released [16].

\section{Statistical combination of $H \rightarrow \gamma \gamma, H \rightarrow Z Z^{\star} \rightarrow \ell \ell \ell \ell, H \rightarrow W W^{\star} \rightarrow \ell \ell v v, H \rightarrow \tau \tau$ and $H \rightarrow b \bar{b}$ decay channels}

Results of statistical combinations of the $H \rightarrow \gamma \gamma, H \rightarrow Z Z^{\star} \rightarrow \ell \ell \ell \ell$ and $H \rightarrow W W^{\star} \rightarrow$ $\ell \ell v v$ decay channels are published in Ref.[7] and Ref.[8]; in some cases preliminary results from Ref.[17] are presented in the note including also $H \rightarrow \tau \tau$ and $H \rightarrow b \bar{b}$.

\subsection{Signal strength, reconstructed mass and measured couplings}

The local probability $p_{0}$ for a background-only experiment to be more signal-like than the observation as a function of $m_{H}$ for the combination of all channels is shown in Fig. $8(\mathrm{a})$, The observed 


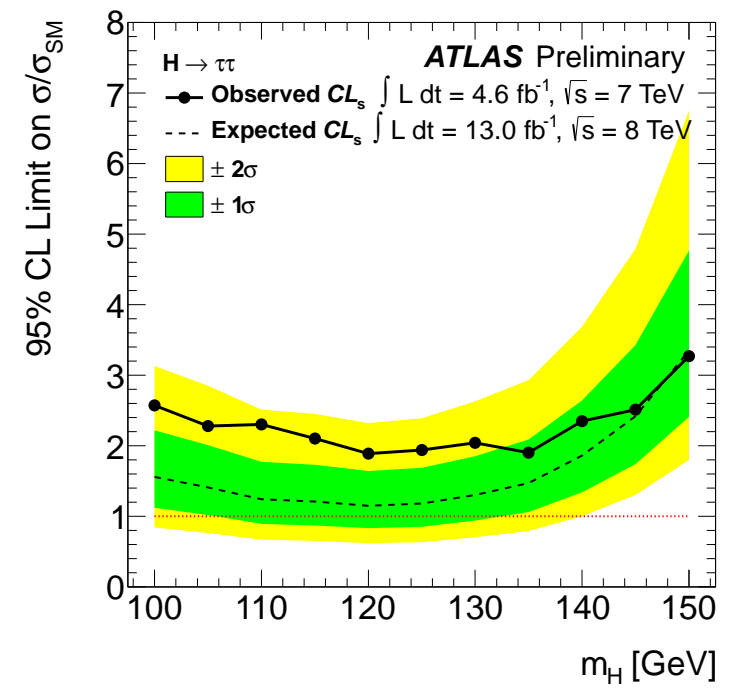

(a)

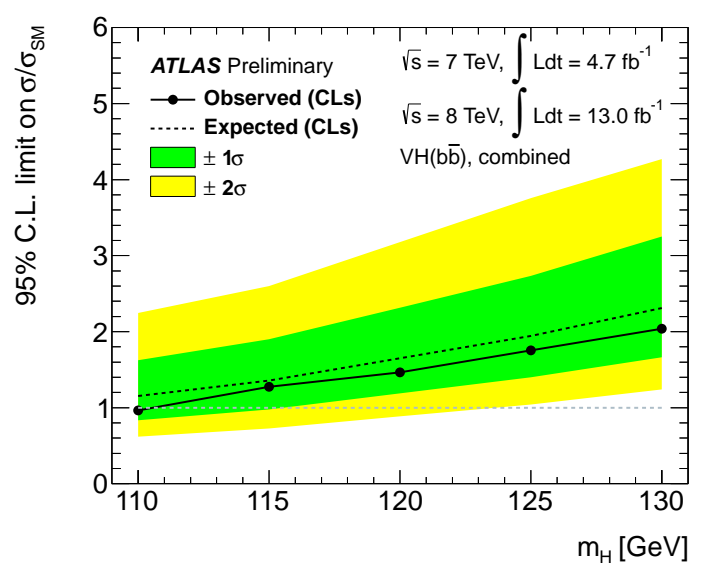

(b)

Figure 7: Observed (solid) and expected (dashed) 95\% CL upper limits on the $\sigma_{H} B R$, normalised to the SM expectation, as a function of $m_{H}$. Expected limits are given for the scenario with no signal. The bands around the dashed line indicate the $1 \sigma$ and $2 \sigma$ uncertainties of the expected limit. (a) $H \rightarrow \tau \tau$ decay channel [13] (b) $V H, H \rightarrow b \bar{b}$ channel [15].

local significance is $10 \sigma$ and the expected significance at $m_{H}=125 \mathrm{GeV}$ is $7.5 \sigma$ [17]. The measured signal mass based on the $H \rightarrow \gamma \gamma$ and $H \rightarrow Z Z^{\star} \rightarrow \ell \ell \ell \ell$ channels is $m_{H}^{o b s}=125.5 \pm 0.2 \pm 0.6$ $\mathrm{GeV}$ with $2.4 \sigma$ tension between the values obtained from these separate channels. The observed signal strength for each of the five mentioned channels, $i$, in units of the SM, defined as $\mu_{i}=$ $\left(\sigma_{H} \times B R_{i}\right)_{\text {meas }} /\left(\sigma_{H} \times B R_{i}\right)_{S M}$, at this mass value is shown in Fig.8(b). The overall signal strength is measured to be $1.30 \pm 0.13$ (stat) \pm 0.14 (sys) at the mass of $125.5 \mathrm{GeV}$ [17]. ${ }^{6}$

Since several Higgs boson production modes are available at the LHC, results shown in twodimensional plots require either some $\mu_{i}$ to be fixed or several $\mu_{i}$ to be related. No direct $t \bar{t} H$ production has been observed yet, hence a common signal strength scale factor $\mu_{g g F+t t H}$ has been assigned to both gluon fusion production ( $g g F)$ and the very small $t \bar{t} H$ production mode, as they both scale dominantly with the $t t H$ coupling in the SM. Similarly, a common signal strength scale factor $\mu_{V B F+V H}$ has been assigned to the $\mathrm{VBF}$ and $V H$ production modes, as they scale with the $W H / Z H$ gauge coupling in the SM. The measurements in the four channels, $H \rightarrow \gamma \gamma$, $H \rightarrow W W^{\star} \rightarrow \ell \ell v v, H \rightarrow Z Z^{\star} \rightarrow \ell \ell \ell \ell$ and $H \rightarrow \tau \tau$, as well as the observed combined ratio $\mu_{V B F+V H} / \mu_{g g F+t t H}=1.2_{-0.5}^{+0.7}$ are compatible with the SM expectation of unity. The $p$-value ${ }^{7}$ when testing the hypothesis $\mu_{V B F+V H} / \mu_{g g F+t t H}=0$ is $0.05 \%$, corresponding to a significance against the vanishing vector boson mediated production assumption of $3.3 \sigma$. The ratio $\mu_{V B F} / \mu_{g g F+t t H}$,

\footnotetext{
${ }^{6}$ The updated overall signal strength based on only the three mentioned bosonic channels is measured to be $1.33 \pm 0.14$ (stat) \pm 0.15 (sys) 䜣.

${ }^{7}$ The $p$-value and significance are calculated for the test hypothesis $\mu_{V B F+V H} / \mu_{g g F+t t H}=0$ against the one-sided alternative $\mu_{V B F+V H} / \mu_{g g F+t t H}>0$ using the profile likelihood test statistic.
} 
Table 1: Expected and observed $p_{0}$ values for the $0^{+}$and $2^{+}$hypotheses as function of the $f_{q \bar{q}}$ of the spin-2 production mechanism in the combined $H$ study [ 8 ]. The values are calculated for the combination of the three $H$ decay channels. The CLs values are also presented.

\begin{tabular}{c||c|c||c|c||c}
\hline \hline$f_{q \bar{q}}$ & $\begin{array}{c}2^{+} \text {assumed } \\
\text { exp. } p_{0}\left(J^{P}=0^{+}\right)\end{array}$ & $\begin{array}{c}0^{+} \text {assumed } \\
\text { exp. } p_{0}\left(J^{P}=2^{+}\right)\end{array}$ & $\begin{array}{c}\text { obs. } p_{0} \\
\left(J^{P}=0^{+}\right)\end{array}$ & $\begin{array}{c}\text { obs. } p_{0} \\
\left(J^{P}=2^{+}\right)\end{array}$ & $\begin{array}{c}C L_{s} \\
\left(J^{P}=2^{+}\right)\end{array}$ \\
\hline $100 \%$ & $3.0 \times 10^{-3}$ & $8.8 \times 10^{-5}$ & 0.81 & $1.6 \times 10^{-6}$ & $0.8 \times 10^{-5}$ \\
$75 \%$ & $9.5 \times 10^{-3}$ & $8.8 \times 10^{-4}$ & 0.81 & $3.2 \times 10^{-5}$ & $1.7 \times 10^{-4}$ \\
$50 \%$ & $1.3 \times 10^{-2}$ & $2.7 \times 10^{-3}$ & 0.84 & $8.6 \times 10^{-5}$ & $5.3 \times 10^{-4}$ \\
$25 \%$ & $6.4 \times 10^{-3}$ & $2.1 \times 10^{-3}$ & 0.80 & $0.9 \times 10^{-4}$ & $4.6 \times 10^{-4}$ \\
$0 \%$ & $2.1 \times 10^{-3}$ & $5.5 \times 10^{-4}$ & 0.63 & $1.5 \times 10^{-4}$ & $4.2 \times 10^{-4}$ \\
\hline \hline
\end{tabular}

where the signal strength $\mu_{V H}$ of the $V H$ Higgs production process is profiled instead of being treated together with $\mu_{V B F}$, gives the same result of $\mu_{V B F} / \mu_{g g F+t t H}=1.2_{-0.5}^{+0.7}$. The $p$-value for $\mu_{V B F} / \mu_{g g F+t t H}=0$ is $0.09 \%$ corresponding to a significance against the vanishing VBF production assumption of $3.1 \sigma$.

In another approach the dependence on the individual production $\mu_{i}$ cancels out when taking the ratio of $\mu_{i} \times B R$ within the same production mode. For the example of the $H \rightarrow \gamma \gamma$ and $H \rightarrow Z Z^{\star} \rightarrow \ell \ell \ell \ell$ channels, this results in a ratio of relative branching ratios $\rho$, defined as: $\rho_{X X / Y Y}=\left(B R(H \rightarrow X X) B R_{S M}(H \rightarrow Y Y)\right) /\left(B R(H \rightarrow Y Y) B R_{S M}(H \rightarrow X X)\right)$. The best-fit values are: $\rho_{\gamma \gamma / Z Z}=1.1_{-0.3}^{+0.4}, \rho_{\gamma \gamma / W W}=1.7_{-0.5}^{+0.7}$ and $\rho_{Z Z / W W}=1.6_{-0.5}^{+0.8}$, in agreement with the SM expectation of one.

The compatibility of the measured yields for the studied channels with the prediction for the SM Higgs boson is tested under various benchmark assumptions probing salient features of the couplings. A summary of all coupling scale factor measurements in all benchmark models is presented in [17] and updated in [7]. For the different tested benchmarks the compatibility with the SM Higgs expectation ranges between $5 \%$ and $10 \%$; hence, no significant deviation from the SM prediction is observed in any of the fits performed.

\subsection{Spin combination}

Three $H$ decay channels are combined to study spin properties of the observed new boson: $H \rightarrow \gamma \gamma, H \rightarrow Z Z^{\star} \rightarrow \ell \ell \ell \ell$ and $H \rightarrow W W^{\star} \rightarrow e v \mu v$ [8]. The complete 8-TeV dataset is used for this study; for the $H \rightarrow Z Z^{\star} \rightarrow \ell \ell \ell \ell$ channel all data obtained at $7 \mathrm{TeV}$ are added. Table 1 shows the expected and observed $p_{0}$ values for the $0^{+}$and $2^{+}$hypotheses for the combination of mentioned channels. The results are shown as function of the $f_{q \bar{q}}$ of the spin-2 production fraction. Data strongly favour the $0^{+}$hypothesis. The $2^{+}$hypothesis is excluded with CL $>99.9 \%$ independent of the assumed contribution of gg-fusion and $q \bar{q}$-annihilation process.

It should be noted that $1^{+}$and $1^{-}$hypotheses were also tested [8]. Related results are not discussed in the note. 


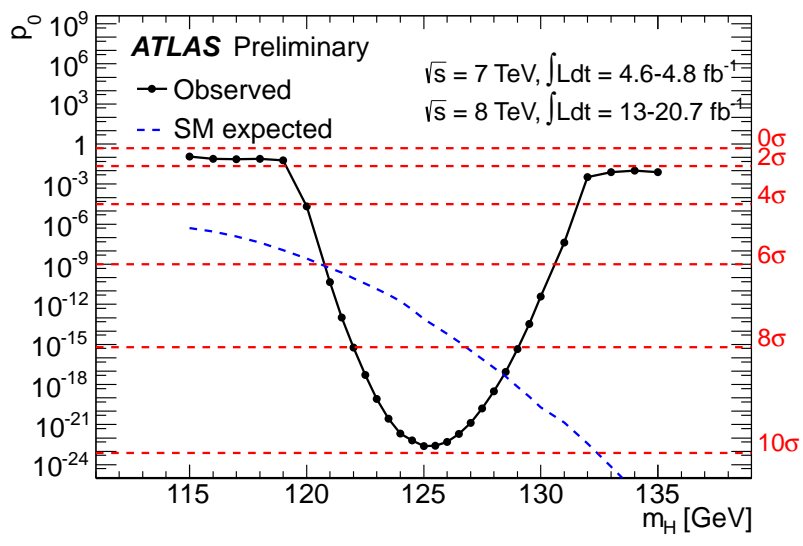

(a)

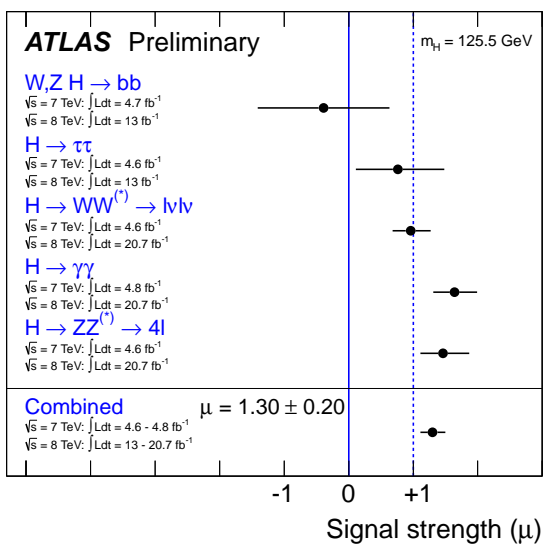

(b)

Figure 8: (a) The local probability $p_{0}$ for a background-only experiment to be more signal-like than the observation as function of $m_{H}$ for the combination of five $H$ channels [17]. The effect of mass scale systematic uncertainties is taken into account by an extrapolation of the sum of a chi-squared and a falling exponential to the distribution of the likelihood ratio-based test statistic for a large number of pseudo-experiments [ $\beta$ ] The dashed curve shows the median expected local $p_{0}$ under the hypothesis of a SM Higgs boson production at that mass. (b) Measurements of the signal strength parameter $\mu$ for $m_{H}=125.5 \mathrm{GeV}$ for the individual channels and their combination [17].

\section{Rare decay channels: $H \rightarrow \mu \mu$ and $H \rightarrow Z \gamma$}

Based on the complete 8-TeV dataset, ATLAS obtained first limits on $H$ production in rare decay channels $H \rightarrow \mu \mu$ and $H \rightarrow Z \gamma$ which are briefly discussed below.

\section{1 $H \rightarrow \mu \mu$ channel [18]}

The search is performed in the region $110-150 \mathrm{GeV}$, where $B R(H \rightarrow \mu \mu)$ is the largest, but it is as small as $\mathrm{O}\left(10^{-4}\right)$. For this reason the expected $\sigma_{H} \times B R$ is only $4 \mathrm{fb}$ at $\sqrt{s}=8 \mathrm{TeV}$ for $m_{H}=125 \mathrm{GeV}$. The signature is two opposite-sign reconstructed isolated muons with $m_{\mu \mu}$ close to $m_{H}$. Background, measured from data using sidebands, is huge and comes mostly from Drell-Yan processes, so excellent invariant-mass resolution is very important; the expected ATLAS detector resolution is $F W H M \approx 5 \mathrm{GeV}$. The $95 \%$ CL exclusion limit lies between $4 \sigma_{S M}$ and $22 \sigma_{S M}$ in the $m_{H}$ range considered, being at $9.8 \sigma_{S M}$ at $125 \mathrm{GeV}$.

\section{2 $H \rightarrow Z \gamma$ channel [19]}

The search mass range is $120-150 \mathrm{GeV}$, where $B R(H \rightarrow Z \gamma)$ is maximal being $\mathrm{O}\left(10^{-3}\right)$. To improve the $\mathrm{S} / \mathrm{B}$ ratio, only di-lepton (electron or muon) $Z$ decays are considered. The resulting cross-section is a few fb at $\sqrt{s}=8 \mathrm{TeV}$ for $m_{H}=125 \mathrm{GeV}$ as in the $H \rightarrow \mu \mu$ case. The signature is an isolated photon together with two opposite-sign reconstruted high- $p_{T}$ isolated muons or electrons from $Z$ decay. The invariant-mass of this $\gamma \ell \ell$-system should be close to $m_{H}$. Background, measured from data using sidebands, is significant. It comes mostly from $Z+\gamma$ production (82\%) 
and $Z+$ jets production (17\%), if a photon is misidentified as a jet. The 95\% CL exclusion limit lies between $6 \sigma_{S M}$ and $36 \sigma_{S M}$ in the $m_{H}$ range considered, being at $18.2 \sigma_{S M}$ at $125 \mathrm{GeV}$.

\section{Conclusion}

In conclusion:

- Based on analysis of $H \rightarrow \gamma \gamma, H \rightarrow Z Z^{\star} \rightarrow \ell \ell \ell \ell, H \rightarrow W W^{\star} \rightarrow \ell \ell v v$ decay channels in complete dataset taken at $7 \mathrm{TeV}$ and $8 \mathrm{TeV}$ together with $H \rightarrow b \bar{b}$ and $H \rightarrow \tau \tau$ channels at 7 $\mathrm{TeV}$ only, the ATLAS experiment discovered a neutral boson with a mass $\approx 125 \mathrm{GeV}$ having a production cross section compatible with the SM Higgs boson; fermionic decay modes of this boson are not yet discovered;

- First measurements of the new boson couplings were performed and they are all in agreement with the SM predictions; coupling to bosons were measured with much better precision than couplings to fermions;

- Different spin/parity hypotheses of the new particle were tested and the one predicted by the $\mathrm{SM}, J^{P}=0^{+}$hypothesis, has strong preference;

- ATLAS is continuing to study properties of the discovered boson and plan to improve the measurements with new data expected starting from year 2015.

After the first long shutdown in 2013-2014 the LHC energy will increase to $\sqrt{s}=13-14 \mathrm{TeV}$, with luminosities around $10^{34} \mathrm{~cm}^{-2} \mathrm{~s}^{-1}$. In anticipation of the high luminosity (HL-LHC) upgrades, the LHC will install equipment in a one-year shutdown in 2018 to allow the instantaneous luminosity to be doubled. An integrated luminosity around $300 \mathrm{fb}^{-1}$, the goal for the approved LHC programme, should be reached by about 2021 . At this point the LHC luminosity can be improved further. After 2022, LHC can be expected to reach luminosities around $5 \times 10^{34} \mathrm{~cm}^{-2} \mathrm{~s}^{-1}$. The ATLAS detector upgrade programme foresees replacement of critical components to allow such operation with a similar detector performance as at present. The total integrated luminosity foreseen for this HL-LHC phase is $3000 \mathrm{fb}^{-1}$. An overview of the expected measurement precision with high luminosity at $\sqrt{s}=14 \mathrm{TeV}$ in each channel for the signal strength and its coupling constants with respect to the SM $H$ with $m_{H}=125 \mathrm{GeV}$ is given in Ref.[20].

\section{References}

[1] L. Evans and P. Bryant (editors), LHC Machine, JINST 3 (2008) S08001.

[2] LHC Higgs Cross Section Working Group, S. Dittmaier et al, Handbook of LHC Higgs Cross Sections: 1. Inclusive Observables arXiv:1101.1593

[3] ATLAS Collaboration, Observation of a new particle in the search for the Standard Model Higgs boson with the ATLAS detector at the LHC, Phys. Lett. B 716 (2012) 1, [arXiv: 1207.7214 ]

[4] CMS Collaboration, Observation of a new boson at a mass of $125 \mathrm{GeV}$ with the CMS experiment at the LHC, Phys. Lett. B 716 (2012) 30, [arXiv: 1207 . 7235]

[5] ATLAS Collaboration, The ATLAS Experiment at the CERN Large Hadron Collider, JINST 3 (2008) S08003. 
[6] J. Allison et al., IEEE Trans. Nucl. Sci. 53 (2006) 270.

S. Agostinelli et al., GEANT4: A Simulation toolkit, Nucl. Instrum. Meth. A 506 (2003) 250.

[7] ATLAS Collaboration, Measurements of Higgs boson production and couplings in diboson final states with the ATLAS detector at the LHC, Phys. Lett. B 726 (2013) 88.

[8] ATLAS Collaboration, Evidence for the spin-0 nature of the Higgs boson using ATLAS data, Phys. Lett. B 726 (2013) 120.

[9] ATLAS Collaboration, Observation and study of the Higgs boson candidate in the two photon decay channel with the ATLAS detector at the LHC, ATLAS-CONF-2012-168 (December 2012), http://cds.cern.ch/record/1499625

[10] ATLAS Collaboration, Measurements of the properties of the Higgs-like boson in the two photon decay channel with the ATLAS detector using $25 \mathrm{fb}^{-1}$ of proton-proton collision data, ATLAS-CONF-2013-012 (March 2013), http://cds.cern.ch/record/1523698

[11] ATLAS Collaboration, Measurements of the properties of the Higgs-like boson in the four lepton decay channel with the ATLAS detector using $25 \mathrm{fb}^{-1}$ of proton-proton collision data, ATLAS-CONF-2013-013 (March 2013), http://cds.cern.ch/record/1523699

[12] ATLAS Collaboration, Measurements of the properties of the Higgs-like boson in the $W W^{\star} \rightarrow \ell \ell v v$ decay channel with the ATLAS detector using $25 \mathrm{fb}^{-1}$ of proton-proton collision data, ATLAS-CONF-2013-030 (March 2013), http://cds.cern.ch/record/1527126

[13] ATLAS Collaboration, Search for the Standard Model Higgs boson in $H \rightarrow \tau^{+} \tau^{-}$decays in proton-proton collisions with the ATLAS detector, ATLAS-CONF-2012-160 (November 2012), http://cds.cern.ch/record/1493624

[14] ATLAS Collaboration, Evidence for Higgs Boson Decays to the $\tau^{+} \tau^{-}$Final State with the ATLAS Detector, ATLAS-CONF-2013-095 (November 2013), http://cds.cern.ch/record/1561970

[15] ATLAS Collaboration, Search for the Standard Model Higgs boson produced in association with a vector boson and decaying to bottom quarks with the ATLAS detector, ATLAS-CONF-2012-161 (November 2012), http://cds.cern.ch/record/1493625

[16] ATLAS Collaboration, Search for the bb decay of the Standard Model Higgs boson in associated (W/Z)H production with the ATLAS detector, ATLAS-CONF-2013-079 (July 2013), http://cds.cern.ch/record/1563235

[17] ATLAS Collaboration, Combined coupling measurements of the Higgs-like boson with the ATLAS detector using up to $25 \mathrm{fb}^{-1}$ of proton-proton collision data, ATLAS-CONF-2013-034 (March 2013), http://cds.cern.ch/record/1528170

[18] ATLAS Collaboration, Search for the Standard Model Higgs boson in $H \rightarrow \mu^{+} \mu^{-}$decays with the ATLAS detector, ATLAS-CONF-2013-010 (March 2013), http://cds.cern.ch/record/1523695

[19] ATLAS Collaboration, Search for the Standard Model Higgs boson in the $H \rightarrow Z \gamma$ decay mode with pp collisions at $\sqrt{s}=7$ and $8 \mathrm{TeV}$, ATLAS-CONF-2013-009 (March 2013), http://cds.cern.ch/record/1523683

[20] ATLAS Collaboration, Projections for measurements of Higgs boson cross sections, branching ratios and coupling parameters with the ATLAS detector at a HL-LHC, ATLAS-PHYS-PUB-2013-014 (October 2013), https://cds.cern.ch/record/1611186 